> Parmi les maladies mitochondriales, un certain nombre sont caractérisées par une instabilité du génome mitochondrial qui se manifeste par la présence de délétions multiples ou de déplétions de ce génome. Ces anomalies sont responsables d'un dysfonctionnement de la chaîne respiratoire mitochondriale, principale source d'énergie indispensable à l'activité cellulaire, et concourent à l'apparition de symptômes plus ou moins graves. Les délétions multiples de l'ADNmt engendrent des atteintes cliniques spécifiques de l'adulte tandis que les cas les plus sévères sont liés aux déplétions de l'ADNmt. Ces deux anomalies possèdent un dénominateur commun : leur spécificité tissulaire. <

\section{Instabilité du génome mitochondrial et pathologies associées}

Emmanuelle Sarzi, Agnès Rötig

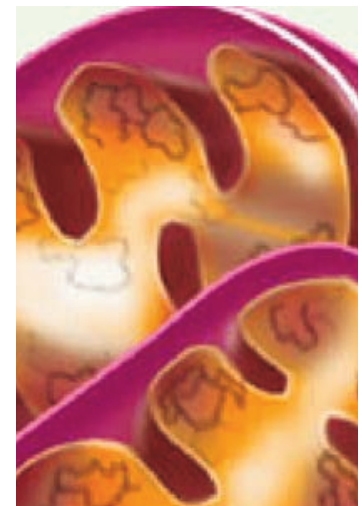

Inserm U781, Hôpital Necker Enfants malades, 149 , rue de Sèvres, 75015 Paris, France. emmanuelle.sarzi@inserm.fr
Les maladies mitochondriales sont des maladies génétiques dues à un dysfonctionnement de la chaîne respiratoire. Elles sont caractérisées par une importante hétérogénéité clinique et génétique. Compte tenu de la double origine des protéines constituant les mitochondries, ces maladies peuvent être dues à des anomalies localisées dans des gènes portés par l'ADN mitochondrial (ADNmt) ou dans des gènes nucléaires. Ces altérations sont responsables in fine d'une perturbation de la production d'énergie au niveau de la chaîne respiratoire mitochondriale. Les délétions multiples et les déplétions de l'ADNmt sont la conséquence de mutations survenues dans des gènes portés par l'ADN nucléaire. L'ensemble de ces gènes détient un rôle direct ou indirect dans le maintien du génome mitochondrial. Ils codent pour des protéines impliquées dans sa réplication ou encore dans la synthèse mitochondriale ou cytosolique des dNTP (dinucléotides triphosphates).

\section{Maladies mitochondriales associées à des délétions multiples de l'ADNmt}

Les maladies mitochondriales liées à la présence de délétions multiples de l'ADNmt sont majoritairement à transmission autosomique dominante et se manifestent le plus souvent à l'âge adulte. La taille et les bornes des délétions sont variables d'un individu à l'autre au sein d'une même famille. D'une manière générale, ces maladies se caractérisent par une ophtalmoplégie externe progressive ${ }^{1}$ (PEO pour progressive external ophthalmoplegia) souvent associée à un ptosis, une faiblesse progressive des muscles squelettiques ainsi qu'une intolérance à l'effort. Les délétions multiples associées à une PEO sont exclusivement retrouvées dans les muscles squelettiques des patients.

\section{Protéines intervenant}

dans la réplication mitochondriale

Trois gènes codant pour des protéines intervenant dans la réplication de l'ADNmt sont impliqués dans les PEO avec délétions multiples de I'ADNmt: POLGI et $P O L G 2$, codant pour les deux sous-unités $\alpha$ et $\beta$ de I'ADN polymérase $\gamma$, et PEOI codant pour I'hélicase mitochondriale Twinkle (Figure 1). On connaît des mutations dominantes et récessives dans le gène POLG1. Leur identification a permis de montrer que les mutations dominantes étaient localisées dans le domaine polymérase, alors que les mutations récessives touchaient à la fois le domaine «spacer» et le domaine exonucléase. Le spectre clinique associé

${ }^{1}$ Ophtalmoplégie : affection caractérisée par une faiblesse progressive des muscles moteurs des globes oculaires et du releveur de la paupière supérieure (source orphanet). 


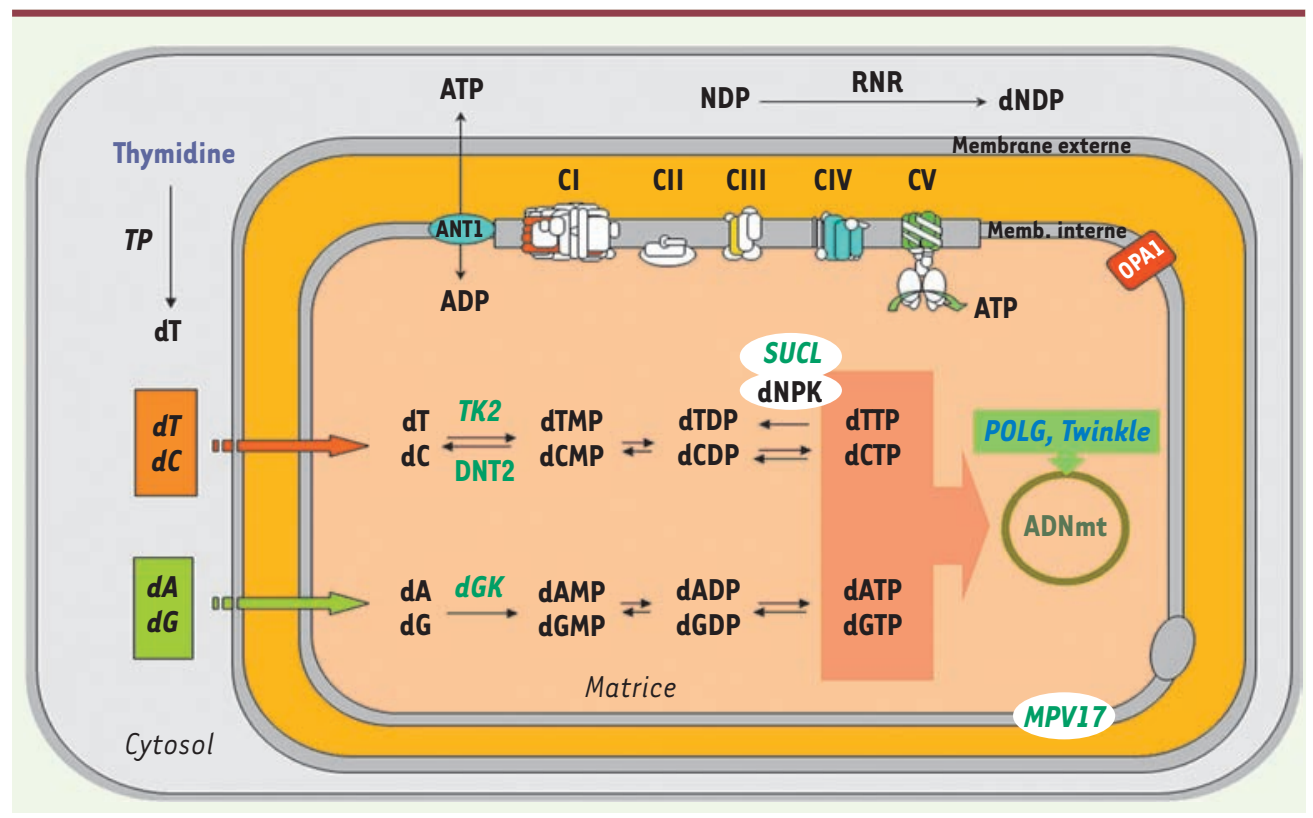

Figure 1. Gènes impliqués dans les délétions multiples et/ou les déplétions de I'ADNmt. Cl, CII, CIII, CIV et CV : complexe I, II, III, IV et V de la chaîne oxydative mitochondriale. $d T, d C, d A, d G$ : désoxythymidine, désoxycytidine, désoxyadénosine, désoxyguanosine; dTMP, dCMP, dAMP, dGMP : désoxynucléotides monophosphate; dTDP, $\mathrm{dCDP}, \mathrm{dADP}, \mathrm{dGDP}$ : désoxynucléotides diphosphate; dTTP, dCTP, dATP, dGTP : désoxynucléotides triphosphate.

à l'ensemble des mutations de POLGI s'étend de la PEO classique à une PEO à laquelle viennent s'ajouter d'autres traits phénotypiques de type ataxique, des symptômes psychiatriques (dépression) voire de type parkinsonien $[1,2]$. Des études in vitro ont permis de montrer que ces délétions multiples seraient dues à un piétinement de la réplication de l'ADNmt issu de la déficience de l'activité polymérase [3]. L’hypothèse selon laquelle les délétions pourraient survenir durant la réparation de l'ADNmt altéré a été proposée mais sa validation nécessiterait des investigations plus poussées [4]. À l'heure actuelle, dans le cas du gène POLG2, une mutation dominante a été décrite associée à une P\&O et à des délétions multiples de I'ADNmt [5] (Figure 1). Les analyses biochimiques ont permis de révéler que cette mutation était responsable d'un défaut d'interaction entre la sous-unité $\alpha$ et la sousunité $\beta$ de la polymérase, empêchant de ce fait la sous-unité $\alpha$ de lier l'ADNmt [5]. Le phénotype associé aux mutations du gène $P E O 1$ se calque sur celui lié aux mutations dans le gène POLGl, à l'exception près que dans le cas de $P E O 1$, seules des mutations dominantes ont été décrites comme étant à l'origine de délétions multiples [6-8]. De récentes études in vitro ont permis de déceler des défauts plus ou moins importants de l'hexamérisation et de l'activité hélicase de la protéine Twinkle en fonction de la mutation testée $[8,9]$. Dernièrement, l'hypothèse d'une réparation anormale des cassures double-brin de l'ADNmt a été avancée pour expliquer les délétions multiples de l'ADNmt engendrées par des mutations dans le gène PEOl [4].

\section{Protéines de la membrane interne mitochondriale}

\section{Mutations du gène SLC25A4}

Parmi les protéines de la membrane interne, on retrouve la protéine ANTl (adénine nucléotide translocase type 1), codée par le gène SLC25A4 ${ }^{2}$ dont la présence de mutations dominantes sont aussi responsables d'une P\&O associée à des délétions multiples de l'ADNmt. Cette protéine participe à la structure du pore de transition de la perméabilité membranaire de la mitochondrie, autrement appelé PTP (permeability transition pore), et assure la translocation de I'ATP et de I'ADP à travers la membrane interne mitochondriale (Figures 1 et 2). Le lien entre la fonction d'ANTl et la formation de délétions multiples de l'ADNmt n'est pas clair. Cependant, les mutations dans SLC25A4 pourraient entraîner un déséquilibre du pool des désoxynucléotides triphosphates ou dNTP entre le cytosol et la mitochondrie [10] (Figure 2). Les dNTP sont les composants essentiels de l'ADN, qu'il soit nucléaire ou mitochondrial. Les dNTP sont présents sous quatre formes: la désoxyadénosine triphosphate (dATP), la désoxycytidine triphosphate (dCTP), la désoxyguanosine triphosphate (dGTP) et enfin la désoxythymidine triphosphate (dTP). Il est important de préciser que la mitochondrie ne possède pas de synthèse de novo de dNTP. Elle importe les dNTP directement du cytosol ou bien les synthétise à l'aide de précurseurs désoxynucléotidiques présents dans le cytosol ( $d A, d T, d G$ et $d C$ ) ou provenant des produits de dégradation de l'ADNmt (Figure 2). Ce recyclage est qualifié de voie de récupération mitochondriale des dNTP. On comprend ainsi pourquoi un déséquilibre des pools de dNTP dans la matrice mitochondriale ou le cytosol peut altérer l'efficacité et la fidélité de la réplication mitochondriale. Une autre hypothèse concerne la pathogénicité de ces mutations: par leur implication dans le fonctionnement du pore de transition de la perméabilité membranaire, les mutations dans le gène SLC25A4 pourraient déclencher une apoptose médiée par la mitochondrie [11].

${ }^{2}$ SLC25A4 : solute carrier family 25 (mitochondrial carrier; adenine nucleotide translocator), member 4. 


\begin{tabular}{|c|c|c|c|c|c|c|}
\hline Fonction & Gènes & Transmission & $\begin{array}{l}\text { Anomalie } \\
\text { de I'ADNmt }\end{array}$ & $\begin{array}{l}\text { Tissu } \\
\text { atteint }\end{array}$ & $\begin{array}{l}\text { Atteinte } \\
\text { clinique }\end{array}$ & $\begin{array}{l}\text { Début de } \\
\text { la maladie }\end{array}$ \\
\hline \multirow{7}{*}{ Réplication mitochondriale } & \multirow{3}{*}{ POLG1 } & Dominant & Délétions multiples & Muscle & PEO & Adulte \\
\hline & & \multirow{2}{*}{ Récessif } & Déplétion & Foie & SDM/Alpers & Enfance \\
\hline & & & Déplétion & Cervelet & MIRAS & Adulte \\
\hline & POLG2 & Dominant & Délétions multiples & Muscle & PEO & Adulte \\
\hline & \multirow{3}{*}{ PE01 } & Dominant & Délétions multiples & Muscle & PEO & Adulte \\
\hline & & \multirow{2}{*}{ Récessif } & Déplétion & Foie & SDM & Enfance \\
\hline & & & Déplétion & Cerveau & IOSCA & Enfance \\
\hline \multirow{9}{*}{$\begin{array}{l}\text { Directe ou indirecte sur le pool } \\
\text { de dNTP mitochondrial } \\
\text { ou cytoplasmique }\end{array}$} & ANTI & Dominant & Délétions multiples & Muscle & PEO & Adulte \\
\hline & ТУМР & Récessif & Délétions/déplétions & Muscle & MNGIE & Adulte \\
\hline & TK2 & Récessif & Déplétion & Muscle & SDM & Enfance \\
\hline & DGUOK & Récessif & Déplétion & Foie & SDM & Enfance \\
\hline & \multirow{3}{*}{ RRM2B } & Récessif & Déplétion & Muscle & SDM & Enfance \\
\hline & & Récessif & Déplétion & Muscle & MNGIE & Adulte \\
\hline & & Dominant & Délétions multiples & Muscle & PEO & Adulte \\
\hline & SUCLA2 & Récessif & Déplétion & Muscle & SDM & Enfance \\
\hline & SUCLGI & Récessif & Déplétion & Muscle et foie & SDM & Enfance \\
\hline $\begin{array}{l}\text { Fusion mitochondriale, } \\
\text { organisation des crêtes, } \\
\text { contrôle de l'apoptose }\end{array}$ & OPA1 & Dominant & Délétions multiples & Muscle & $\mathrm{AOD}+$ & Adulte \\
\hline Inconnue & MPV17 & Récessif & Déplétion & Foie & SDM & Enfance \\
\hline
\end{tabular}

Tableau I. Gènes impliqués dans l'apparition de délétions multiples et/ou de déplétions de l'ADNmt. PEO : progressive external ophthalmoplegia ; SDM : syndrome de déplétion de l'ADNmt ; MIRAS : mitochondrial recessive ataxia syndrome; MNGIE : mitochondrial neuro-gastro-intestinal encephalopathy ; $A O D+$ : atrophie optique dominante «plus ».

\section{Mutations du gène OPA1}

Le défaut d'une autre des protéines de la membrane interne, codée par le gène OPAl (optic atrophy l), est aussi responsable de délétions multiples de l'ADNmt [12]. Ce gène code pour une protéine à activité GTPase de la famille des dynamines qui est impliquée dans la fusion mitochondriale, le maintien du potentiel de membrane mitochondrial, l'organisation des crêtes et le contrôle de l'apoptose [13] (Figure 1). On sait depuis des années que les nombreuses mutations tronquantes dans ce gène sont responsables de la survenue d'une atrophie optique dominante isolée pouvant conduire à une cécité complète. Récemment, l'implication de nouvelles mutations de ce gène a été démontrée dans une atrophie optique syndromique associée à une ataxie, une PEO ou une surdité $[12,14]$. Les patients présentent des délétions multiples de l'ADNmt dans les cellules des muscles squelettiques. Contrairement aux mutations tronquantes conduisant à une haplo-insuffisance, les mutations responsables de délétions multiples de l'ADNmt sont toutes des mutations faux-sens dans le domaine GTPase de la protéine. Ces mutations confèreraient un gain ou une perte de fonction à la protéine OPAl. Comme précédemment, les mécanismes pouvant expliquer l'apparition de délétions multiples de l'ADNmt ne sont pas très clairs. Cependant, plusieurs hypothèses ont été émises : des perturbations de l'activité GTPase pourraient affecter le pool des dNTP ; la protéine OPAl, du fait de sa présence dans la membrane interne, pourrait, quand elle est mutée, déstabiliser l'ancrage de I'ADNmt par un changement conformationnel des crêtes mitochondriales ou via une interaction directe avec le domaine amino-terminal de la protéine OPAl.

\section{Protéines impliquées}

dans le maintien du pool de dNTP cytosolique

Des protéines cytoplasmiques jouent un rôle dans le maintien du génome mitochondrial : c'est le cas de la thymidine phosphorylase (TP) codée par le gène TYMP (Figures 1 et 2). Les mutations réces- 


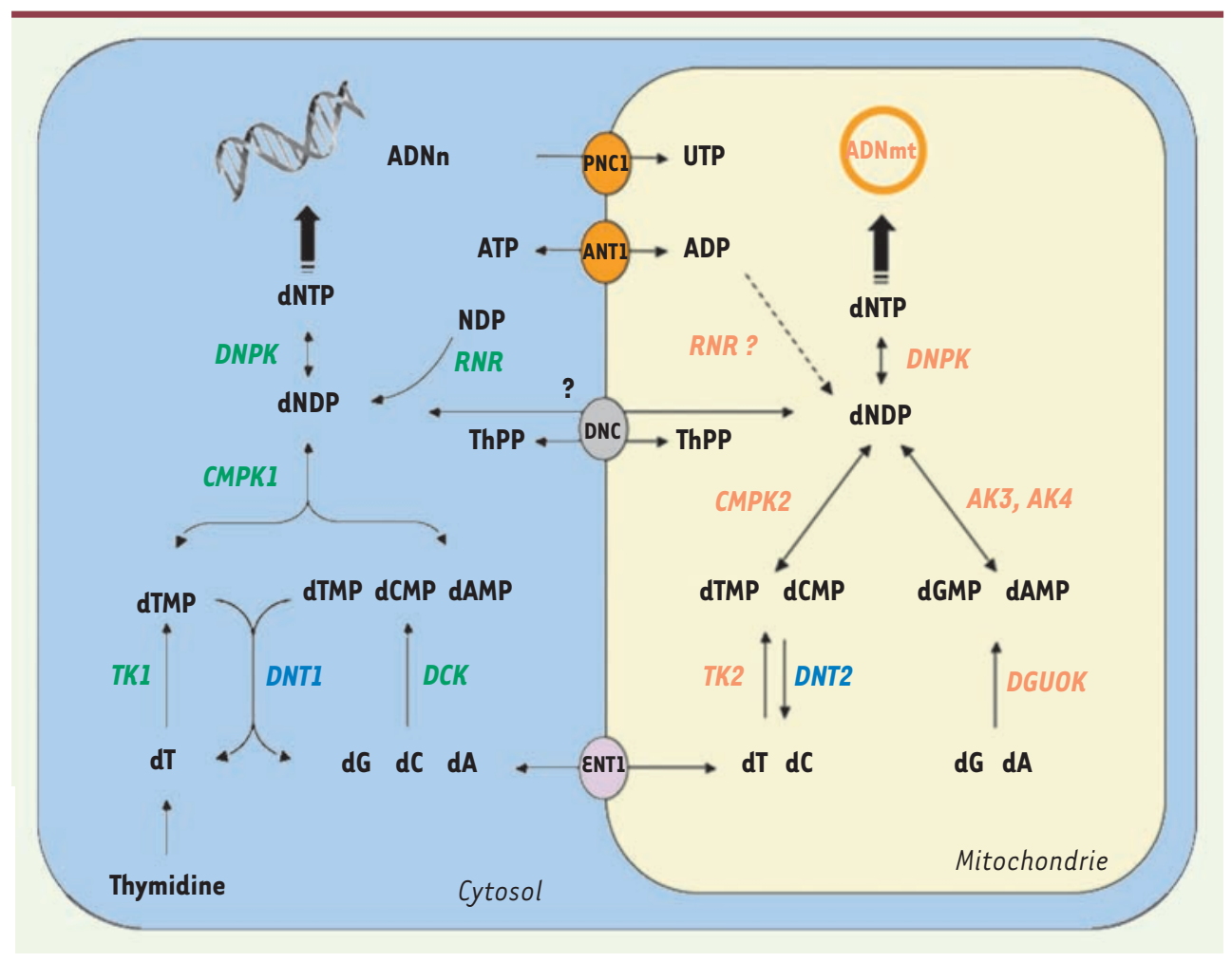

Figure 2. Métabolisme des dNTP. Ce schéma décrit les différentes étapes cytosoliques et mitochondriales de la synthèse des dNTP. dNTP : désoxynucléotide triphosphate; dATP, dCTP, dGTP, dTTP : désoxyadénosine triphosphate, désoxycytidine triphosphate, désoxyguanosine triphosphate, désoxythymidine triphosphate; dNDP: désoxynuclotide diphosphate, dNPK : désoxynucléotide phosphate kinase; $d A, d G, d T, d C$ : désoxynucléotides; TK : thymidine kinase ; DGUOK : désoxyguanosine kinase; AK: adénylate kinase; DNT2 : désoxypyrimidines nucléotidase; RNR : ribonucléotide réductase. CMPK : cytidine monophosphate kinase; DNC: désoxynucléotide carrier; ThPP : thiamine pyrophosphate. ADNn : acide désoxyribonucléique nucléaire.

sives trouvées dans ce gène sont responsables du syndrome MNGIE (mitochondrial neuro-gastro-intestinal encephalopathy) marqué par la coexistence de délétions multiples et de déplétions de I'ADNmt. L'accumulation de thymidine dans le cytoplasme, en déséquilibrant le pool des dNTP, entraînerait en effet une instabilité de l'ADNmt de ces patients [15]. Dernièrement, une mutation dominante a été décrite dans le gène $R R M 2 B$ codant pour la petite sous-unité $\mathrm{p} 53 \mathrm{R} 2$ de la ribonucléotide réductase (RNR). Elle est responsable d'une PEO associée à des délétions multiples de l'ADNmt [16] (Figures 1 et 2). La ribonucléotide réductase est indispensable pour la synthèse des dNTP cytosoliques et son activité dans les cellules postmitotiques dépendrait de la présence de la protéine p53R2. C'est une nouvelle fois un déséquilibre du pool des dNTP qui serait responsable de l'instabilité du génome mitochondrial observée [17] (Tableau I).

\section{Maladies mitochondriales associées à des déplétions de l'ADNmt}

Tout d'abord, il est important de rappeler que de nombreuses copies d'ADNmt sont présentes dans les mitochondries. Le syndrome de déplétion de l'ADNmt se traduit par une diminution marquée du nombre de copies d'ADNmt causée par des mutations autosomiques récessives de gènes nucléaires et par un tableau clinique sévère apparaissant dans les premiers mois de la vie et conduisant à un décès précoce.

\section{Déplétion de l'ADNmt et anomalies de réplication de l'ADNmt}

Des mutations récessives du gène POLGl codant pour une protéine de la machinerie réplicative sont associées au syndrome
d'Alpers-Huttenlocher (ASH, OMIM \#203700) [18]. Ce dernier, contrairement à la PEO décrite ci-dessus qui est une maladie de l'adulte, se manifeste assez tôt dans l'enfance et conduit à un décès prématuré des patients. Elle débute par une encéphalopathie précoce caractérisée par un retard psychomoteur, une épilepsie récalcitrante et une atteinte hépatique. Les patients présentent des déplétions de l'ADNmt dans le foie (taux d'ADNmt résiduel inférieur à $25 \%$ du taux normal), le muscle (moins de $30 \%$ du taux normal) et le cortex frontal (moins de $70 \%$ du taux normal). Les mutations observées dans le syndrome d'Alpers sont localisées majoritairement dans les domaines dits «spacer» et polymérase de I'ADN polymérase $\gamma$ (POLG). Plus de 120 mutations du gène POLGI ont été répertoriées ${ }^{3}$. Comme précédemment, la perturbation de la réplication de l'ADNmt serait à l'origine des déplétions de l'ADNmt [3]. Dernièrement, le syndrome MIRAS (mitochondrial recessive ataxia) lié à la présence d'une mutation récessive spécifique du gène $P O L G I$ a été associé à une diminution du nombre de copies d'ADNmt dans le cerveau des patients atteints [19]. Des mutations récessives du gène codant pour l'hélicase Twinkle conduisent également à des déplétions de l'ADNmt (moins de $15 \%$ d'ADNmt résiduel) alors que les mutations dominantes sont associées à des PEO (voir ci-dessus) [20].

\footnotetext{
http://tools.niehs.nih.gov/polg/
} 
Les patients porteurs de mutations récessives ont une atteinte hépatique précoce, un retard de croissance et psychomoteur ainsi que des crises convulsives tardives conduisant au décès vers l'âge de 2 à 3 ans. Une telle présentation clinique n'est pas sans rappeler celle des patients atteints du syndrome d'Alpers causé par des mutations récessives du gène POLG1. Ainsi, le tableau clinique des patients présentant des mutations récessives dans le gène $P E O 1$ associées à une déplétion de l'ADNmt dans le foie a été à juste titre qualifié de syndrome d'Alpers-like [21]. Des déplétions de l'ADNmt ont également été décrites dans le cerveau (taux résiduel d'ADNmt inférieur à $20 \%$ du taux normal) de patients présentant un syndrome IOSCA (pour infantile-onset spinocerebellar ataxia) lié à une mutation récessive spécifique du gène $P E O 1$ [19].

\section{Déplétion de l'ADNmt et anomalies}

de la voie de récupération des dNTP mitochondriaux

La voie de récupération mitochondriale des dNTP est très semblable à la voie cytosolique mais elle fait intervenir des enzymes spécifiques de la mitochondrie (Figure 1) [22, 23]. Le déficit enzymatique touchant la désoxyguanosine kinase ( $d G K$ ) et la thymidine kinase 2 (TK2), assurant la première étape de la voie de récupération des purines et des pyrimidines, entraîne un défaut de synthèse des dNTP et, par conséquent, conduit à un nombre très limité de molécules d'ADNmt. Il est important de noter que, tout comme les délétions multiples, les déplétions de l'ADNmt ont une spécificité tissulaire. En effet, les mutations dans le gène DGUOK conduisent à une déplétion de l'ADNmt dans le foie (taux d'ADNmt inférieur à $10 \%$ du taux normal) associée à une atteinte hépatocérébrale alors que les mutations du gène TK2 entraînent une déplétion dans le muscle squelettique (taux d'ADNmt inférieur à $15 \%$ du taux normal) et une myopathie (Figures 1 et 2).

Un autre aspect du syndrome de déplétion de I'ADNmt lié à un déséquilibre du pool des dNTP a été illustré par la découverte de mutations dans le gène SUCLA2 codant pour la sous-unité $\beta$ de la succinyl-CoA ligase (SUCL) [24]. La pathologie associée à ces mutations se caractérise par une atteinte multisystémique où prédominent un syndrome encéphalomyopathique et une acidémie méthylmalonique. La déplétion de I'ADNmt a été mise en évidence dans le muscle squelettique (taux résiduel d'ADNmt de 15 à $40 \%$ ). La SUCL est une enzyme de la matrice mitochondriale qui fait partie du cycle de Krebs. Elle assure la synthèse du succinylCoA à partir du succinate qui va s'accumuler en cas de déficit de cette enzyme et être converti en acide méthylmalonique puis excrété dans les urines. Le lien entre ce défaut d'activité et la présence de déplétion de l'ADNmt n'a pas été clairement établi. Cependant, il a été montré, chez le rat, que la succinyl-CoA ligase coprécipitait avec la nucléoside diphosphate kinase dNPK [25]. Cela suggère que les mutations trouvées dans le gène SUCLA2 pourraient modifier l'interaction de la protéine SUCL avec la dNPK, conduisant à un défaut de synthèse des dNTP dans la voie de récupération mitochondriale. Des mutations dans un second gène, le gène SUCLG1, codant pour la sous-unité $\alpha$ de la SUCL, ont été récemment identifiées chez des patients présentant une acidose lactique néonatale suivie d'un décès précoce [26]. La déplétion de l'ADNmt s'est révélée à la fois dans le muscle ( $15 \%$ du taux normal) et dans le foie ( $60 \%$ du taux normal), et trouverait elle aussi son origine dans la diminution de l'activité de la dNPK résultant de son incapacité à interagir avec la SUCL (Figures 1 et 2).

\section{Déplétion de l'ADNmt et anomalies}

\section{de la synthèse des dNTP cytosoliques}

Des mutations dominantes dans le gène $R R M 2 B$ (ribonucleotide reductase M2 B [TP53 inducible]) sont responsables de délétions multiples de I'ADNmt. Des mutations récessives de ce même gène avaient été identifiées précédemment comme responsables de déplétions sévères de l'ADNmt (taux résiduel de moins de $5 \%$ ) dans le muscle squelettique chez des patients présentant une encéphalomyopathie et une tubulopathie [27]. Les mutations récessives conduisent à un décès précoce des patients dans les premières semaines de vie. Très récemment, deux mutations pathogènes du gène $R R M 2 B$ ont été décrites dans un cas de syndrome MNGIE habituellement lié à des mutations du gène TYMP (voir ci-dessus) [28]. Ces mutations ont pour conséquence une déplétion de l'ADNmt dans le muscle squelettique (taux résiduel d'ADNmt de moins de $12 \%$ ).

Enfin, le syndrome hépatocérébral de déplétion de l'ADNmt peut également être dû à des mutations récessives dans le gène MPVI7 [29]. Ce gène code pour une protéine de la membrane interne de la mitochondrie dont la fonction reste encore inconnue (Figure 1). Les mutations du gène MPVI7 sont responsables de déplétions hépatiques sévères en ADNmt ( 5 à $15 \%$ de taux résiduel).

\section{Conclusion}

Les anomalies qualitatives et quantitatives de l'ADNmt que nous venons de détailler sont marquées par leur spécificité tissulaire alors que les gènes impliqués dans ces maladies ont tous une expression ubiquitaire. Aujourd'hui, cette spécificité tissulaire n'est toujours pas comprise. Elle pourrait trouver son origine dans la variabilité d'expression de ces gènes ainsi que dans celle d'autres gènes impliqués dans la même voie métabolique. Autre hypothèse, l'intervention de modifications post-traductionnelles des protéines exprimées par ces gènes. Mais ceci reste entièrement à prouver. Enfin, le fait que des mutations dominantes ou récessives d'un même gène puissent engendrer des anomalies quantitatives ou qualitatives de I'ADNmt pourrait être lié au taux résiduel de protéine fonctionnelle. En effet, la présence de protéines inactives et/ou tronquées dans le cas de muta- 
tions récessives induirait une perte totale de fonction, tandis qu'une mutation dominante produirait, par haplo-insuffisance, des protéines défectueuses interférant avec leurs homologues sauvages et induisant ainsi une activité protéique résiduelle altérée.

Aucune étude récente ne permet de déterminer la prévalence des anomalies de la maintenance du génome mitochondrial. Actuellement, les déplétions de l'ADNmt représentent $8 \%$ des maladies mitochondriales détectées dans la cohorte de patients que nous avons étudiés ces dernières années à l'hôpital Necker-Enfants malades. II est important de souligner que dans de très nombreux cas, le gène responsable de telles anomalies est encore inconnu. De plus, le nombre important de protéines potentiellement impliquées dans le maintien du génome mitochondrial et l'hétérogénéité clinique et génétique des maladies mitochondriales rendent difficile l'élucidation des bases moléculaires de ces anomalies qualitatives et quantitatives. $\diamond$

\section{SUMMARY}

Mitochondrial genome instability and associated diseases

Some cases of mitochondrial diseases are due to mitochondrial DNA instability: multiple deletions or depletions. These anomalies are responsible for a mitochondrial respiratory chain impairment leading to various clinical involvements ranging from mild features with multiple mtDNA deletions to severe organ failure and premature death caused by mtDNA depletions. Both, deletions and depletions share an important and common feature between these two specificities: indeed, both are expressed in a tissue-specific manner. $\diamond$

\section{REMERCIEMENTS}

À l'AFM (Association française contre les myopathies).

\section{CONFLIT D'INTÉRÊTS}

Les auteurs déclarent n'avoir aucun conflit d'intérêts concernant les données publiées dans cet article.

\section{RÉFÉRENCES}

1. Lamantea $\varepsilon$, Tiranti V, Bordoni A, et al. Mutations of mitochondrial DNA polymerase gammaA are a frequent cause of autosomal dominant or recessive progressive external ophthalmoplegia. Ann Neurol $2002 ; 52: 211-9$.

2. Luoma P, Melberg A, Rinne J0, et al. Parkinsonism, premature menopause, and mitochondrial DNA polymerase gamma mutations: clinical and molecular genetic study. Lancet 2004 ; $364: 875-82$.

3. Wanrooij S, Goffart S, Pohjoismäki JL, et al. Expression of catalytic mutants of the mtDNA helicase Twinkle and polymerase POLG causes distinct replication stalling phenotypes. Nucleic Acids Res 2007 ; $35: 3238-51$

4. Krishnan KJ, Reeve AK, Samuels DC, et al. What causes mitochondrial DNA deletions in human cells? Nat Genet $2008 ; 40: 275-9$.

5. Longley MJ, Clark S, Yu Wai Man C, et al. Mutant POLG2 disrupts DNA polymerase gamma subunits and causes progressive external ophthalmoplegia. Am J Hum Genet 2006 ; 78 : 1026-34.

6. Baloh RH, Salavaggione $\varepsilon$, Milbrandt J, Pestronk A. Familial parkinsonism and ophthalmoplegia from a mutation in the mitochondrial DNA helicase twinkle. Arch Neurol $2007 ; 64: 998-1000$.

7. Hudson G, Deschauer M, Busse K, et al. Sensory ataxic neuropathy due to a novel C100rf2 mutation with probable germline mosaicism. Neurology $2005 ; 64: 371-3$.

8. Spelbrink JN, Li FY, Tiranti V, et al. Human mitochondrial DNA deletions associated with mutations in the gene encoding Twinkle, a phage 77 gene 4-like protein localized in mitochondria. Nat Genet $2001 ; 28: 223-31$.
9. Korhonen JA, Pande V, Holmlund T, et al. Structure-function defects of the TWINKLE linker region in progressive external ophthalmoplegia. J Mol Biol 2008 ; $377: 691-705$.

10. Kaukonen J, Juselius JK, Tiranti V, et al. Role of adenine nucleotide translocator 1 in mtDNA maintenance. Science $2000 ; 289: 782-5$.

11. Bauer MK, Schubert A, Rocks 0 , Grimm S. Adenine nucleotide translocase-1, a component of the permeability transition pore, can dominantly induce apoptosis. J Cell Biol 1999 ; 147 : 1493-502.

12. Amati-Bonneau P, Valentino ML, Reynier P, et al. OPAl mutations induce mitochondrial DNA instability and optic atrophy 'plus' phenotypes. Brain $2008 ; 131: 338-51$.

13. Lenaers $G$, Reynier $P$, Elachouri $G$, et al. OPAl functions in mitochondria and dysfunctions in optic nerve. Int J Biochem Cell Biol 2009 ; $41: 1866-74$.

14. Hudson G, Amati-Bonneau P, Blakely EL, et al. Mutation of OPAl causes dominant optic atrophy with external ophthalmoplegia, ataxia, deafness and multiple mitochondrial DNA deletions: a novel disorder of mtDNA maintenance. Brain 2008 ; 131 : 329-37.

15. Giordano C, Sebastiani M, Plazzi G, et al. Mitochondrial neurogastrointestinal encephalomyopathy: evidence of mitochondrial DNA depletion in the small intestine. Gastroenterology 2006 ; $130: 893-901$.

16. Tyynismaa $H$, Ylikallio $\varepsilon$, Patel $M$, et al. A heterozygous truncating mutation in RRM2B causes autosomal-dominant progressive external ophthalmoplegia with multiple mtDNA deletions. Am J Hum Genet 2009; $85: 290-5$.

17. Bourdon, A, Rotig A. p53R2 : DNA repair or mitochondrial DNA synthesis? Med Sci (Paris) $2007 ; 23: 803-5$.

18. Naviaux RK, Nguyen KV. POLG mutations associated with Alpers' syndrome and mitochondrial DNA depletion. Ann Neurol 2004 ; 55 : 706-12.

19. Hakonen AH, Goffart S, Marjavaara S, et al. Infantile-onset spinocerebellar ataxia and mitochondrial recessive ataxia syndrome are associated with neuronal complex I defect and mtDNA depletion. Hum Mol Genet $2008 ; 17: 3822-35$.

20. Sarzi $\varepsilon$, Goffart $S$, Serre V, et al. Twinkle helicase (PEOl) gene mutation causes mitochondrial DNA depletion. Ann Neurol 2007 ; 62 : 579-87.

21. Hakonen AH, Isohanni P, Paetau A, et al. Recessive Twinkle mutations in early onset encephalopathy with mtDNA depletion. Brain 2007 ; $130: 3032-40$.

22. Mandel H, Szargel R, Labay V, et al. The deoxyguanosine kinase gene is mutated in individuals with depleted hepatocerebral mitochondrial DNA. Nat Genet $2001 ; 29: 337-41$.

23. Saada A, Shaag A, Mandel H, et al. Mutant mitochondrial thymidine kinase in mitochondrial DNA depletion myopathy. Nat Genet 2001 ; $29: 342-4$.

24. Ostergaard $\varepsilon$, Hansen FJ, Sorensen N, et al. Mitochondria encephalomyopathy with elevated methylmalonic acid is caused by SUCLA2 mutations. Brain $2007 ; 130: 853-61$

25. Kowluru A, Tannous M, Chen HQ. Localization and characterization of the mitochondrial isoform of the nucleoside diphosphate kinase in the pancreatic beta cell: evidence for its complexation with mitochondrial succinyl-CoA synthetase. Arch Biochem Biophys $2002 ; 398: 160-9$.

26. Ostergaard $\varepsilon$, Christensen $\varepsilon$, Kristensen $\varepsilon$, et al. Deficiency of the alpha subunit of succinate-coenzyme $A$ ligase causes fatal infantile lactic acidosis with mitochondrial DNA depletion. Am J Hum Genet 2007 ; $81: 383-7$.

27. Bourdon A, Minai L, Serre V, et al. Mutation of RRM2B, encoding p53-controlled ribonucleotide reductase (p53R2), causes severe mitochondrial DNA depletion. Nat Genet 2007 ; 39 : 776-80.

28. Shaibani A, Shchelochkov OA, Zhang S, et al. Mitochondrial neurogastrointestinal encephalopathy due to mutations in RRM2B. Arch Neurol 2009 ; $66: 1028-32$.

29. Spinazzola A, Viscomi C, Fernandez-Vizarra $\varepsilon$, et al. MPV17 encodes an inner mitochondrial membrane protein and is mutated in infantile hepatic mitochondrial DNA depletion. Nat Genet 2006 ; $38: 570-5$.

\section{TIRÉS À PART}

E. Sarzi 